\section{Prevalência e fatores associados ao Transtorno de Estresse \\ Pós-Traumático (TEPT) em profissionais de emergência: uma revisão sistemática da literatura}

\section{Prevalence and factors associated with Posttraumatic Stress Disorder (PTSD) in emergency workers: a systematic literature review}

\section{Eduardo de Paula Lima}

\section{Ada Ávila Assunção}

Programa de Pós-Graduação em Saúde Pública da Faculdade de Medicina da Universidade Federal de Minas Gerais.

Correspondência: Eduardo de Paula Lima. Av. Alfredo Balena, 190 - sala 705, CEP 30310-100 Belo Horizonte, MG. E-mail: edpl@hotmail.com

\section{Resumo}

Objetivo: Identificar as prevalências de Transtorno de Estresse Pós-Traumático (TEPT) em profissionais dos serviços de emergências e conhecer os fatores associados ao desfecho. Métodos: Foram consultadas sete bases de dados (MEDLINE via Pubmed, PsycINFO, LILACS, SciELO, BDENF, DISASTRES e MEDCARIB) entre os dias $10 \mathrm{e}$ 25 de setembro de 2009, restringindo-se a pesquisa a artigos em português, inglês ou espanhol publicados no período de 2004 a 2009. Os descritores utilizados abarcaram termos relacionados a profissionais/serviços de emergência, transtorno de estresse póstraumático e condições de trabalho e saúde ocupacional. Foram incluídos estudos observacionais quantitativos que investigaram a prevalência de TEPT e fatores determinantes ou associados à doença em amostras de bombeiros, profissionais de ambulância, profissionais da Cruz Vermelha e profissionais de emergência em hospitais. Foram excluídos estudos com base em amostras inadequadas para os objetivos da revisão (policiais, voluntários e filhos de profissionais de emergência) ou que não incluíram sintomas de TEPT como variável dependente em pelo menos um dos objetivos. Resultados: A leitura dos títulos e resumos resultou em 30 artigos. A revisão das publicações completas permitiu a inclusão e análise de 17 artigos. Os estudos selecionados adotaram diferentes delineamentos, instrumentos, critérios diagnósticos e técnicas estatísticas de análise. A prevalência da doença variou da ausência de registro de casos à taxa de $38,5 \%$. Características sociodemográficas, biológicas e psicológicas, morbidade, exposição a eventos traumáticos ocupacionais e não-ocupacionais, e características do trabalho e do emprego associaram-se ao TEPT em profissionais de emergência. Conclusão: Variáveis individuais e de contexto são consideradas fatores associados ou de risco para o TEPT e explicitam o caráter mutidimensional do desfecho em foco.

Palavras-chave: Saúde mental. Transtorno de estresse pós-traumático. Fatores de risco. Profissionais de emergência. Epidemiologia. Saúde ocupacional. 


\section{Abstract}

Objective: To identify the prevalence of Posttraumatic Stress Disorder (PTSD) in emergency workers and determine the factors associated with outcomes. Methods: Seven databases were consulted (Medline via Pubmed, PsycINFO, LILACS, SciELO, BDENF, DISASTERS, and MEDCARIB) between September 10 and 25, 2009. The search only included articles published in Portuguese, English or Spanish between 2004 and 2009. The key-words involved terms related to emergency services/workers, Posttraumatic stress disorder, working conditions, and occupational health. Quantitative observational studies on PTSD prevalence and determinant or associated factors regarding the health of firefighters, emergency ambulance personnel, Red Cross workers, and medical emergency workers were included. Studies using samples unrelated to the purposes of this review (police officers, volunteers and emergency workers' children) or that did not include PTSD symptoms as the dependent variable in at least one of the objectives of the study were excluded. Results: 30 articles were selected by reading the titles and abstracts. 17 complete articles were reviewed and analyzed. Studies adopted different research designs, instruments and diagnostic criteria for statistical analysis. The prevalence of the disease ranged from absence of reported cases to a rate of $38.5 \%$. Socio-demographic, biological and psychological characteristics, morbidity, exposure to occupational and non-occupational traumatic events, and work and job features were associated with the prevalence of PTSD in emergency workers. Conclusion: Individual and contextual variables are considered as factors associated with PTSD, which explains the multidimensional nature of the outcome studied.

Keywords: Mental health. Posttraumatic stress disorder. Risk factors. Emergency workers. Epidemiology. Occupational health.

\section{Introdução}

Profissionais de emergência, entre eles bombeiros, profissionais de ambulância e profissionais em hospitais, atuam em contextos que exigem respostas rápidas visando à eficácia dos atendimentos. As situações enfrentadas por esses profissionais freqüentemente são imprevisíveis e de risco para as suas próprias vidas e para as vidas das vítimas socorridas ${ }^{1-4}$. Ademais, os profissionais expostos enfrentam eventos adversos: acidentes, agressões físicas, contato com doenças infecciosas etc.

A exposição constante a eventos ocupacionais adversos pode influenciar negativamente a saúde mental. Entre as possíveis formas de adoecimento mental de trabalhadores em serviços de emergência destaca-se na presente revisão o transtorno de ansiedade ligado à vivência de traumas denominado Transtorno de Estresse PósTraumático - TEPT 5 .

De acordo com a quarta edição revisada do Manual Diagnóstico e Estatístico de Transtornos Mentais - DSM-IV-TR ${ }^{6}$, o diagnóstico da doença é essencialmente clínico e diz respeito à presença de sintomas específicos após a exposição a um evento potencialmente traumático (Critério Al) vivido com medo intenso, horror ou impotência diante da situação (Critério A2).

Quanto aos sintomas, os mais comuns são agrupados em três conjuntos distintos: sintomas de revivecência do evento traumático (Critério B), esquiva de estímulos associados ao trauma e entorpecimento da reatividade geral (Critério $\mathrm{C}$ ) e sintomas de excitabilidade aumentada (Critério D).

O diagnóstico definitivo de TEPT exige ainda a persistência dos sintomas por pelo menos um mês (Critério E), com intensidade suficiente para interferir no funcionamento social, ocupacional ou em outras áreas importantes da vida do indivíduo (Critério F). Vale ressaltar que na literatura se encontra uma distinção entre sintomas de TEPT decorrentes da exposição direta e indireta a eventos traumáticos ${ }^{5,7}$. Esta diferenciação é importante, pois permite 
identificar casos da doença em indivíduos diretamente vitimizados (TEPT primário) e em pessoas que apenas testemunharam eventos traumáticos (TEPT secundário).

Quanto aos fatores associados ao desencadeamento dos sintomas são citados: características sociodemográficas, funcionamento psicológico, morbidade psiquiátrica, tipo e intensidade de exposição à traumas ${ }^{8-12}$.

Em se tratando dos profissionais dos serviços de emergências, por estarem inseridos em contextos específicos, os pesquisadores abordam características distintas daquelas enfocadas na população em geral, a saber: 0 treinamento recebido, a duração e a frequência da exposição aos eventos traumáticos e a influência de variáveis organizacionais ${ }^{7,13-16}$.

Nesta direção, os autores, buscando estabelecer associações entre TEPT e serviços de emergência analisam aspectos em distintos planos, sendo eles os fatores individuais, sociais e ocupacionais ${ }^{2-4,17}$.

Vale mencionar que reações ao estresse grave e transtornos de adaptação são os transtornos mentais mais freqüentes entre brasileiros com contrato formal de trabalho. Em 2008, foram registrados 7.026 casos de reações ao estresse grave, sendo considerado um entre os 50 tipos de doenças/acidentes de trabalho mais freqüentes naquele ano ${ }^{18}$. Contudo, apesar da frequência, ainda são incipientes as investigações sobre TEPT em nosso meio. Estudos envolvendo profissionais de emergência, em especial, são importantes no intuito de avaliar o impacto da exposição ocupacional a eventos traumáticos e identificar fatores de risco para TEPT nestes grupos. Trabalhos anteriores indicam, por exemplo, maiores taxas de prevalência e associação com variáveis ocupacionais ${ }^{7,13-15}$.

O objetivo da presente revisão foi identificar as prevalências de TEPT em profissionais dos serviços de emergências e conhecer os fatores associados ao desfecho.

\section{Método}

\section{Bases e estratégias de busca}

As bases de dados consultadas foram: MEDLINE via Pubmed, PsycINFO, LILACS, SciELO, BDENF, DISASTRES e MEDCARIB. A busca foi realizada entre os dias 10 e 25 de setembro de 2009, restringindo-se a artigos em português, inglês ou espanhol publicados no período de 2004 a 2009.

A seleção dos descritores conjugou listas disponíveis nas bases de dados e os descritores associados a artigos examinados previamente (Quadro 1).

\section{Critérios de seleção}

Foram incluídos nesta revisão artigos completos que preencheram os seguintes critérios:

- disponibilidade dos resumos nas bases de dados;

Quadro 1 - Descritores expandidos de acordo com as indicações encontradas nas bases de dados. Chart 1 - Expanded keywords according to data found in databases.

\begin{tabular}{|c|c|}
\hline $\begin{array}{l}\text { Profissionais/Serviços de } \\
\text { emergência }\end{array}$ & 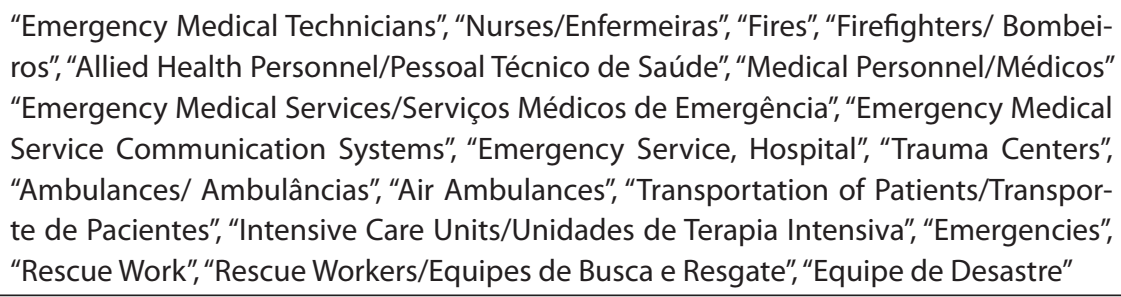 \\
\hline $\begin{array}{l}\text { Transtorno de Estresse } \\
\text { Pós-Traumático (TETP) }\end{array}$ & $\begin{array}{l}\text { "Stress Disorders, Posttraumatic Transtornos de Estresse Traumático", "Stress Disor- } \\
\text { ders, Traumatic, Acute" }\end{array}$ \\
\hline $\begin{array}{l}\text { Condições de trabalho e } \\
\text { saúde ocupacional }\end{array}$ & $\begin{array}{l}\text { "Workload/ Carga de Trabalho", “Occupational Diseases", "Occupational Health/Saúde } \\
\text { Ocupacional", “Workplace", “Occupational Exposure/ Exposição Ocupacional”, “Work } \\
\text { Related IIlness", ,Working Conditions" }\end{array}$ \\
\hline
\end{tabular}


- originalidade;

- uso de metodologia quantitativa;

- amostra composta por bombeiros, profissionais de ambulância (resgate e/ou atendimento pré-hospitalar) ou profissionais de serviços de emergência em hospitais;

- apresentação da prevalência deTEPT ou possibilidade de estimativa de prevalência a partir dos resultados apresentados;

- investigação de fatores determinantes ou associados ao TEPT; e

- presença de sintomas de estresse póstraumático considerada como variável dependente em pelo menos um dos objetivos propostos no artigo.

Foram excluídos os estudos que avaliaram apenas profissionais não contemplados no quarto critério de inclusão descrito acima (policiais e voluntários sem formação específica), artigos interessados em analisar o curso clínico do TEPT e artigos publicados sob a forma de editoriais, entrevistas, projetos e notas clínicas.

\section{Extração dos dados}

Os artigos selecionados foram analisados e registrados em um protocolo construído pelos autores, cuja estrutura foi organizada em oito tópicos:

- introdução;

- objetivos;

- delineamento do estudo;

- definição das variáveis;

- amostra;

- instrumentos de coleta de dados;

- plano de análise dos dados; e

- resultados e discussão.

As variáveis independentes foram agrupadas segundo os seguintes parâmetros: variáveis individuais (características sóciodemográfica, biológicas e psicológicas e morbidade) e variáveis de contexto (exposição a eventos traumáticos ocupacionais e não-ocupacionais e características do trabalho e do emprego).

\section{Resultados}

\section{O processo de revisão}

Foram encontrados 69 artigos potencialmente relevantes considerando a definição das bases de dados (fase 1) e dos descritores de interesse (fase 2). A leitura dos resumos pelos dois autores (fase 3 ) excluiu 39 artigos:

- 24 não apresentaram dados primários ou originais (revisões, editoriais, entrevistas, projetos e notas clínicas);

- 10 artigos estudaram amostras diversas daquelas focalizadas no presente estudo (policiais, voluntários, filhos de profissionais de emergência e moradores e trabalhadores que auxiliaram na reconstrução de locais afetados por desastres);

- dois artigos não apresentaram os resumos;

- dois artigos não utilizaram metodologia quantitativa; e, finalmente,

- um artigo não fez referência à avaliação de sintomas de TEPT.

Trinta artigos foram selecionados nesta etapa. Na etapa final de análise, os trabalhos selecionados foram lidos na íntegra (fase 4). Dois artigos não apresentaram dados de prevalência e doze não incluíram o TEPT como variável dependente, sendo, portanto, excluídos. Os trinta artigos selecionados para análise na etapa final foram localizados. Incluiu-se um único estudo que não tinha sido detectado na busca inicial. Trata-se de um estudo longitudinal (coorte prospectivo) com bombeiros em fase de treinamento ${ }^{19}$. A escolha do artigo se justifica por se tratar de um desdobramento de um estudo previamente incluído na revisão ${ }^{20}$. Finalmente, 17 artigos constituem o corpus da presente revisão conforme ilustra a Figura 1.

Os autores dos estudos revisados buscaram estimar o grau de associação e o poder de predição das variáveis, tanto as de natureza individual quanto àquelas relacionadas ao contexto em relação aos sintomas de estresse pós-traumático. Este artigo está estruturado na mesma direção. Inicialmente 


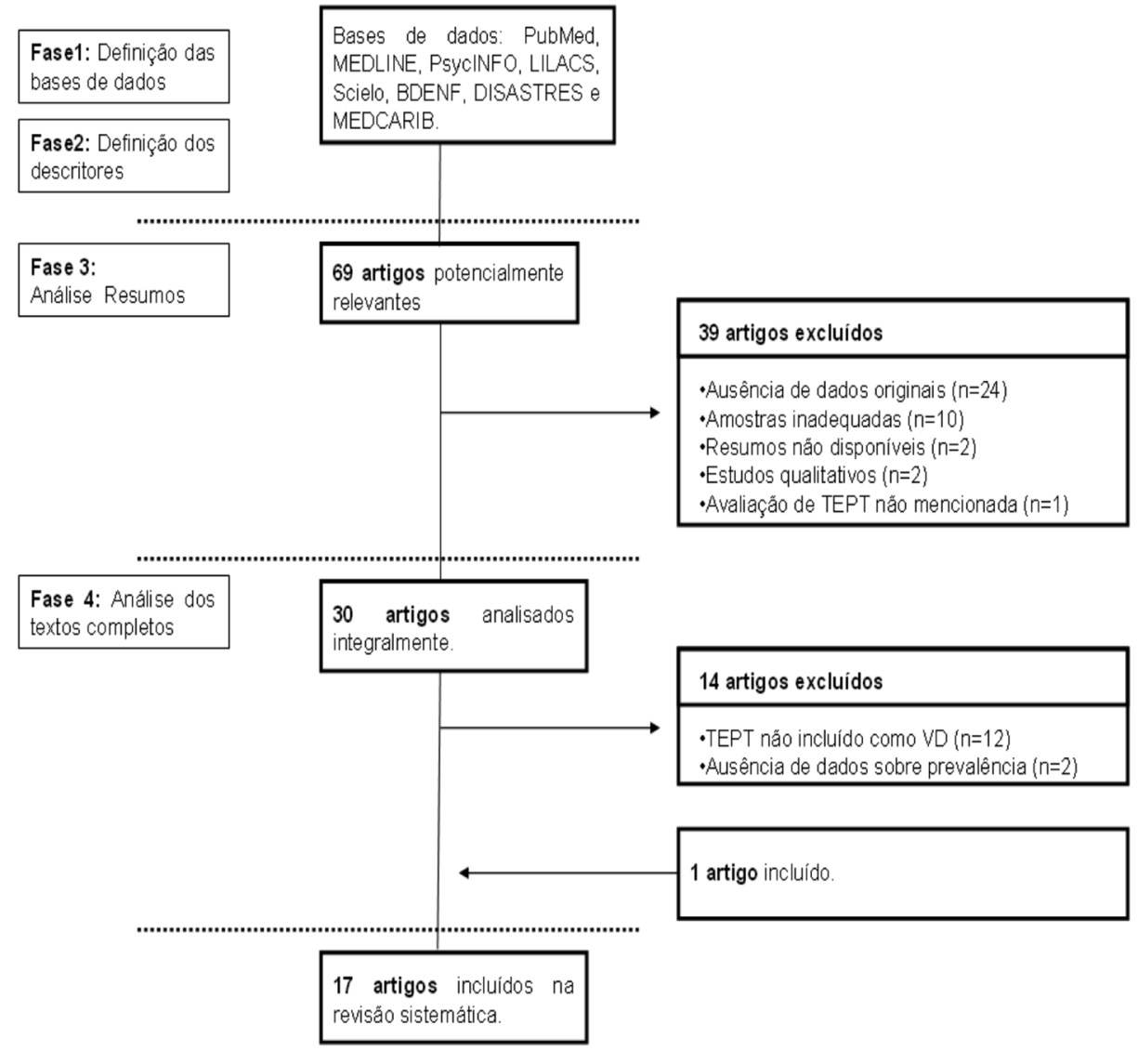

Figura 1 - Esquema representativo dos procedimentos de seleção dos artigos.

Figure 1 - Representation of procedures for selecting articles.

são apresentadas as prevalências encontradas e, a seguir, os resultados, seguindo a natureza (individuais ou contextuais) das associações identificadas.

O primeiro bloco trata dos fatores individuais e inclui características sociodemográficas, biológicas e psicológicas, e morbidade. O segundo bloco diz respeito aos fatores de contexto como variáveis ocupacionais e organizacionais.

As populações estudadas foram as seguintes: bombeiros, profissionais de ambulância, profissionais da cruz vermelha envolvidos em missões de busca e salvamento de vítimas de desastres e profissionais de emergência em hospitais. As prevalências de TEPT encontradas nos referidos grupos variaram entre ausência de registro de casos em bombeiros recém-formados ${ }^{20,21}$, até a taxa de $38,5 \%$ encontrada entre profissio- nais alocados em unidades de tratamento intensivo para vítimas da síndrome respiratória aguda $-\mathrm{SARS}^{22}$. A Tabela 1 expõe as referidas taxas discriminadas por populações específicas e as variáveis associadas ao desfecho em cada um dos 17 estudos.

\section{Fatores individuais}

Onze artigos (11/17) investigaram a relação entre fatores individuais e TEPT. Na Tabela 1 estão apresentados apenas aqueles fatores significativamente associados à presença e intensidade de sintomas deTEPT em todas as análises conduzidas.

\section{Características sociodemográficas}

Cinco estudos (5/17) investigaram a relação entre TEPT e as características so- 
Tabela 1 - Prevalência e fatores associados ao TEPT em profissionais de serviços de emergências segundo tipo de estudo, autores, ano e local.

Table 1 - Prevalence and PTSD associated factors in emergency workers according to type of study, authors, year and site.

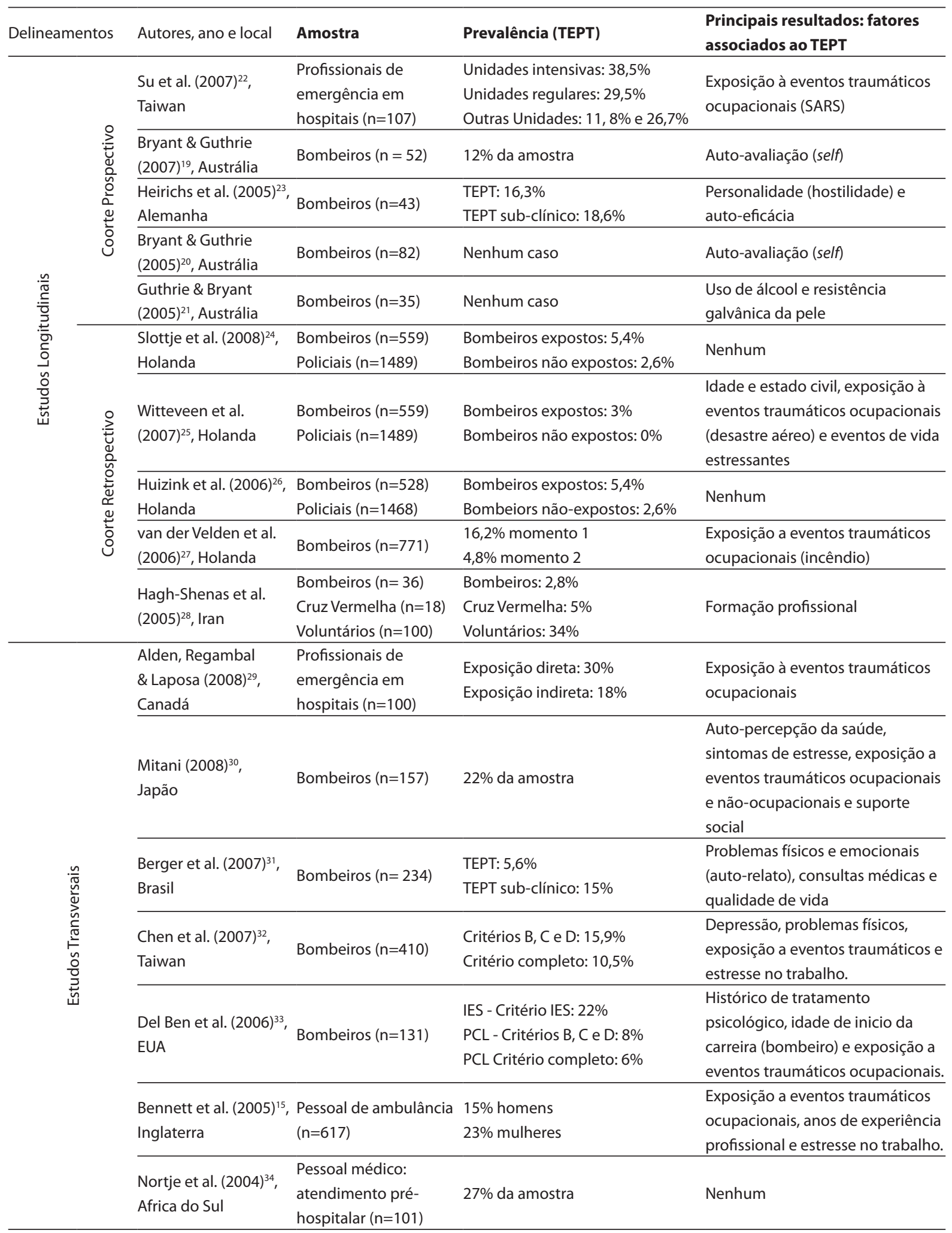


ciodemográficas (idade, sexo, etnia, estado civil, tempo de relacionamento conjugal, número de filhos e escolaridade) ${ }^{15,25,31-33}$. Apenas Witteveen et al. ${ }^{25}$ encontraram associações significativas entre TEPT e as variáveis pesquisadas no estudo longitudinal que avaliou bombeiros e policiais de Amsterdam em evento do tipo desastre aéreo. Os autores buscaram alcançar dois objetivos:

- comparar profissionais expostos e não expostos ao evento em relação à morbidade psiquiátrica e estressores ocupacionais, $\mathrm{e}$

- investigar fatores associados à morbidade psiquiátrica.

A análise de regressão linear realizada adotou os sintomas de TEPT como variável dependente e as características sociodemográficas e o nível de exposição ao evento como variáveis independentes. Encontrouse uma associação positiva entre TEPT e idade, e estado civil solteiro.

\section{Características biológicas}

Dois estudos (2/17) focalizaram as características biológicas, sendo elas medidas psicofisiológicas $^{21}$ e endócrinas ${ }^{23}$. Apenas o estudo longitudinal prospectivo de Guthrie \& Bryant ${ }^{21}$ identificou relações positivas entre variáveis biológicas e TEPT. Os autores do trabalho focaram o impacto psicológico da exposição a eventos traumáticos em 71 bombeiros australianos recém-formados. A fim de avaliar o valor preditivo de medidas psicofisiológicas para sintomas de TEPT após exposição à eventos traumáticos, os sujeitos foram avaliados em duas etapas:

- durante o período de treinamento profissional (linha de base); e

- após 12 meses de prática profissional.

A resposta de sobressalto a estímulos auditivos foi considerada o indicador psicofisiológico, tendo sido avaliada por meio da resistência galvânica da pele e da atividade
EMG do músculo orbicularis oculi. A análise de regressão múltipla permitiu identificar a resistência galvânica da pele como preditora do desfecho investigado, explicando $18 \%$ da variância*. É importante ressaltar que o uso de álcool na linha de base também se mostrou um preditor significativo de TEPT no estudo relatado.

\section{Características psicológicas}

Quatro estudos (4/17) investigaram a relação entre TEPT e características psicológicas, sendo elas as seguintes: personalidade, auto-eficácia, avaliação sobre si mesmo e sobre o mundo, e sentimentos de culpa sobre acontecimentos passados ${ }^{19,20,23,34}$. Foram encontradas associações significativas entre os aspectos citados e TEPT em três artigos.

Dois estudos de delineamento longitudinal prospectivo foram conduzidos em uma amostra de bombeiros em treinamento na brigada de incêndio de New South Wales, Sidnei, Austrália. Os autores publicaram um primeiro estudo com uma amostra de 68 bombeiros $^{20}$ e um segundo, dois anos depois, incluindo uma nova avaliação da mesma amostra $(n=52)^{19}$. Com o objetivo de investigar o valor preditivo para a ocorrência de sintomas de TEPT, padrões disfuncionais de avaliação da realidade presentes antes da exposição a eventos traumáticos foram avaliados em três etapas:

- linha de base: durante o treinamento, antes da exposição ocupacional a eventos traumáticos;

- tempo 2: seis meses após o início da atividade profissional ${ }^{20} ; \mathrm{e}$

- tempo 3: três anos e meio após o início da atividade profissional ${ }^{19}$.

Os padrões disfuncionais de avaliação foram considerados em relação à auto-avaliação (self), conceito semelhante à autoeficácia, à avaliação sobre o mundo e aos sentimentos de culpa sobre eventos ocorridos no passado. Os resultados indicaram

* A resistência galvânica da pele mensura a resistência da pele a uma pequena corrente elétrica e é considerada uma medida autonômica da reação do indivíduo a estímulos externos. 
uma associação positiva significativa entre a auto-avaliação negativa e sintomas de TEPT seis meses (tempo 2) e três anos (tempo 3) após o início da atividade profissional .

O terceiro estudo, também de caráter prospectivo, avaliou 43 bombeiros alemães em fase de treinamento visando identificar fatores de risco para sintomas de TEPT nos sujeitos antes da exposição a eventos traumáticos de origem ocupacional ${ }^{23}$. Os participantes foram estudados em cinco etapas: a primeira avaliação durante a formação profissional (linha de base) e as quatro avaliações subseqüentes em intervalos fixos de 6 meses entre cada uma delas. Na linha de base foram examinados sintomas psicopatológicos, personalidade, auto-eficácia e medidas endócrinas como possíveis variáveis preditoras. Baixa autoeficácia e personalidade (hostilidade) foram bons preditores de TEPT, explicando juntos $42 \%$ da variância observada.

\section{Morbidade}

As morbidades de interesse em seis estudos (6/17) foram avaliadas segundo um leque variado de indicadores, sintomas e diagnósticos, sendo eles os seguintes: sintomas de depressão, ansiedade, estresse e psicossomáticos, obsessividade-compulsividade, sensibilidade interpessoal, idéias paranóides, psicoticismo, alexitimia, fadiga, distúrbios do sono, histórico de tratamento psicológico, número de hospitalizações e consultas médicas, problemas físicos e uso de álcool e tabaco ${ }^{23,25,30-33}$. Quatro estudos (4/17) identificaram relações positivas entre TEPT e problemas físicos e mentais.

O primeiro estudo, de corte transversal, avaliou 157 bombeiros japoneses com o objetivo de investigar associações entre sintomas de TEPT e exposição a eventos traumáticos, auto-percepção da saúde, sintomas de estresse, estressores no trabalho e suporte social $^{30}$. As análises foram conduzidas em três etapas. Primeiro os participantes foram divididos em dois grupos de acordo com risco para TEPT segundo os escores na Japanese-Language Version of Impact
Event Scale (IES-R-J). Na segunda etapa, os participantes considerados com alto risco para TEPT (28/128) foram divididos em dois grupos: expostos e não expostos. Por fim, na última etapa, os participantes expostos a eventos traumáticos foram divididos em dois grupos: expostos com alto risco para TEPT $(n=14)$ e expostos com baixo risco para TEPT $(\mathrm{n}=40)$. Os sujeitos com maior chance de apresentarem TEPT relataram maior comprometimento da saúde e registros de sintomas de estresse. $\mathrm{O}$ autor relatou ainda uma associação positiva entre baixo suporte social e sintomas de TEPT na amostra estudada.

O segundo estudo, de delineamento transversal, avaliou 234 bombeiros alocados em uma unidade de resgate da cidade do Rio de Janeiro, com o objetivo de estimar a prevalência de TEPT e investigar a relação entre a doença e variáveis sociodemográficas, funcionamento psicossocial, saúde física e mental e qualidade de vida ${ }^{31}$. Os participantes foram divididos em três grupos: TEPT clínico, TEPT sub-clínico, e saudáveis. Comparações entre os grupos identificaram: maior freqüência de problemas emocionais auto-relatados e maior número de consultas médicas no ano anterior entre os participantes com TEPT sub-clínico quando comparados aos participantes saudáveis; e maior limitação funcional relacionada a problemas emocionais, pior qualidade de vida geral e maior limitação funcional relacionada a problemas de saúde de ordem física entre os participantes com TEPT clínico quando comparados ao grupo saudável.

O terceiro estudo, de desenho transversal, investigou a qualidade de vida, a prevalência e os fatores associados ao TEPT e depressão em 410 bombeiros da cidade de Kaohsiung em Taiwan ${ }^{32}$. Os participantes foram distribuídos em três grupos: saudáveis, com sintomas subclínicos de TEPT ou depressão, e com sintomas clínicos de TEPT ou depressão. As análises realizadas evidenciaram a presença de sintomas depressivos e de problemas genitais e urinários como variáveis significativamente associadas ao diagnóstico de TEPT. 
O último estudo, de desenho transversal, examinou 131 bombeiros americanos a fim de estimar e comparar a prevalência de TEPT de acordo com os diferentes instrumentos utilizados. Buscou-se também identificar fatores determinantes de adoecimento $^{33}$. Foram realizadas três análises de regressão múltipla adotando os sintomas de TEPT como variável resposta e distintos grupos de variáveis explicativas. A primeira análise considerou características sociodemográficas, funcionamento psicossocial e variáveis relacionadas ao trabalho como possíveis variáveis explicativas (análise 1). A segunda incluiu diferentes aspectos da exposição a eventos traumáticos como possíveis preditores (análise 2). Por fim, na última análise (análise 3) o autores incluíram todas as variáveis consideradas boas preditoras nas análise de regressão anteriores. Os resultados indicaram uma associação positiva entre histórico de tratamento psicológico anterior e TEPT.

\section{Fatores do contexto}

Onze trabalhos (11/17) investigaram a relação entre variáveis de contexto e TEPT. Na Tabela 1 estão apresentadas apenas as variáveis significativamente associadas à presença e intensidade de sintomas deTEPT em todas as análises conduzidas.

\section{Exposição a eventos traumáticos ocupacionais e não-ocupacionais}

A exposição a eventos traumáticos foi avaliada em 10 estudos (10/17), tomando diferentes indicadores como intensidade, freqüência e impacto do evento. Catástrofes, eventos presentes no cotidiano de serviços de emergências, e eventos traumáticos não relacionados ao trabalho foram estudados. Cinco artigos (5/17) focalizaram a exposição de profissionais de emergência a catástrofes ${ }^{22,24-27}$, sendo três com resultados significativos.

Su et al. ${ }^{22}$ delinearam um estudo epidemiológico prospectivo em Taiwan, tendo como grupo alvo as enfermeiras atuando em unidades de atendimento à pacientes vítimas da epidemia de SARS. A avaliação dos participantes foi realizada logo após o início do funcionamento das unidades de atendimento às vítimas da doença (linha de base) e semanalmente durante as quatro semanas subseqüentes. "O objetivo dos autores foi identificar determinantes de adoecimento e comparar a morbidade psiquiátrica em quatro grupos de profissionais":

- 44 enfermeiras em unidades de tratamento regular de SARS

- 26 enfermeiras em unidades de tratamento intensivo de SARS

- 17 enfermeiras em unidades de pacientes com problemas cardíacos

- 15 enfermeiras em unidades de pacientes neurológicos

Os resultados indicaram que sentimentos negativos em relação à epidemia diagnosticados na linha de base foram preditores significativos de TEPT.

O segundo estudo, citado anteriormente, focando uma catástrofe vivenciada por bombeiros e policiais expostos a um desastre aéreo em Amsterdam identificou uma relação entre sintomas de TEPT e o grau de exposição dos profissionais ao evento ${ }^{25}$. O grau de exposição foi definido como o número e o tipo de tarefas desempenhadas no local do acidente. A análise de regressão hierárquica identificou o grau de exposição como um bom preditor da doença. Os autores identificaram ainda o número de eventos de vida estressantes como um preditor significativo de TEPT.

O terceiro estudo, de delineamento longitudinal, investigou bombeiros holandeses envolvidos em um desastre. O evento principal em questão ocorreu em 2000, quando uma fábrica de fogos explodiu na cidade de Enshede deixando mortos, feridos e desabrigados. O artigo analisou o valor preditivo de diferentes eventos traumáticos para a saúde dos profissionais ${ }^{27}$. Os autores compararam sintomas de depressão, ansiedade e TEPT em expostos $(\mathrm{n}=639)$ e não expostos $(\mathrm{m}=$ 132) ao desastre. Os resultados mostraram que os participantes expostos apresentaram 
maior intensidade de sintomas de intrusão e esquiva (TEPT). A frequência de exposição a eventos traumáticos rotineiros também foi considerada preditora significativa no modelo de regressão.

Eventos traumáticos presentes no cotidiano de serviços de emergências e eventos traumáticos não relacionados ao trabalho foram estudados em seis artigos $(6 / 17)^{15,27,29,30,32,33}$, todos com resultados significativos.

Os estudos, já citados, realizados na Holanda $^{27}$, Japão ${ }^{30}$, Taiwan ${ }^{32}$, e EUA ${ }^{33}$ indicaram que eventos traumáticos cotidianos presentes nos serviços de emergência estão significativamente associados ao TEPT. O estudo de Del Ben et al. ${ }^{33}$, em particular, identificou uma relação negativa entre TEPT e atendimento ocorrências mistas não específicas (controle de trânsito, falsas chamadas, entre outras), indicando que eventos ocupacionais não-traumáticos estão associados a uma menor intensidade de sintomas. Além disso, os autores encontraram uma relação positiva entre intensidade de sentimentos de horror diante de eventos traumáticos e TEPT .

Bennet et al. ${ }^{15}$ investigaram a contribuição de aspectos individuais e ocupacionais para o adoecimento psíquico de 617 profissionais pertencentes ao serviço de ambulância britânico, incluindo profissionais da saúde não médicos e técnicos em emergência. Os autores encontraram associações positivas significativas entre TEPT e estressores vinculados ao atendimento de vítimas, como acidentes envolvendo crianças, pessoas queimadas e cadáveres humanos.

Por fim, Alden et al. ${ }^{29}$ apresentaram o único estudo que avaliou tanto exposição direta quanto indireta e suas associações com os sintomas de TEPT em profissionais de emergências. Os autores estudaram 100 profissionais de serviços de emergência em hospitais do Canadá. O objetivo do estudo transversal foi comparar a intensidade e o padrão de sintomas de TEPT entre profissionais que foram diretamente expostos a eventos traumáticos e profissionais que apenas presenciaram tais tipos de eventos no contexto ocupacional. Os resultados não indicaram diferenças significativas em relação à gravidade de sintomas nos dois grupos. Entretanto, o perfil de sintomas variou: o grupo exposto diretamente a traumas apresentou sintomas mais graves de excitabilidade, quando comparado aos sujeitos do outro grupo.

\section{Características do trabalho e do emprego}

Diferentes aspectos do trabalho foram considerados em 5 estudos (5/17), os quais focalizaram: formação profissional, número de empregos, idade de ingresso na profissão, satisfação e estresse no trabalho ${ }^{15,28,30,32,33}$. Quatro estudos encontraram associações significativas com TEPT.

Os estudos, já citados, realizados em Taiwan e EUA indicaram uma associação entre TEPT e o nível de estresse expresso na percepção sobre a carga de trabalho e sobre a avaliação da performance nas tarefas realizadas $^{32} \mathrm{e}$ a idade de início da carreira ${ }^{33}$.

No artigo, também já mencionado, realizado com profissionais ingleses ${ }^{15}$, os autores encontraram resultados positivos utilizando duas estratégias distintas de análise dos dados: uma análise de regressão linear múltipla incluindo apenas os profissionais que relataram dificuldades de memória no mês anterior e a intensidade de sintomas de TEPT como variável dependente; e uma análise de regressão logística incluindo todos os participantes do estudo e o diagnóstico de TEPT como variável dependente dicotômica. Na análise de regressão linear a intensidade de estressores organizacionais (conflitos entre colegas, falsas chamadas dirigidas ao serviço de ambulância etc.) e o maior número de anos de experiência profissional foram considerados bons preditores do desfecho. No modelo de regressão logística, apenas a intensidade dos estressores organizacionais foi preditora de TEPT.

Por fim, pesquisadores iranianos investigaram a relação entre a formação profissional de indivíduos envolvidos em atividades de resgate e sintomas de TEPT ${ }^{28}$. Os autores 
buscaram comparar a intensidade de sintomas psicopatológicos entre profissionais de resgate com experiência e treinamento para realização das atividades e voluntários sem formação em serviços de salvamento. O grupo de profissionais contou com 31 bombeiros e 18 profissionais pertencentes à Cruz vermelha. O grupo de voluntários foi composto por 100 estudantes universitários. Ambos os grupos estiveram envolvidos em um terremoto na província de Kerman, Iran. A comparação dos índices de prevalência de TEPT entre os três grupos indicou que um número significativamente maior de estudantes voluntários desenvolveu a doença, quando comparados aos profissionais dos outros dois grupos.

\section{Discussão}

Esta revisão buscou apreender a prevalência de TEPT e os fatores associados ao desfecho em 17 artigos selecionados sob critérios elaborados pelos autores. O conjunto dos artigos evidenciou diferentes taxas de prevalência, tendo avaliado fatores de natureza distinta, individuais ou de contexto, implicados no desenvolvimento de TEPT. Comprovou-se a adoção de delineamentos do tipo transversal ou longitudinal, utilizando-se de diferentes instrumentos, critérios diagnósticos da doença e técnicas estatísticas de análise dos dados. Apesar de a ampla maioria focalizar bombeiros e profissionais das emergências médicas, outros profissionais foram alvo das pesquisas. Diante dessa heterogeneidade, há limites para as inferências quanto à magnitude $\mathrm{e}$ fatores associados ao desencadeamento de TEPT.

O exame do delineamento do estudo foi crucial para a identificação das características, tanto individuais quanto contextuais, preditoras e associadas ao TEPT. Fatores associados ao TEPT em estudos transversais não se confirmam em estudos longitudinais ${ }^{23}$. As evidências de causalidade são mais fortes em estudos longitudinais prospectivos, pois os fatores de risco são avaliados em uma etapa anterior ao possível desfecho. Estudos retrospectivos se mostraram adequados para a investigação de causa e efeito, mas estão sujeitos a possíveis vieses, como memória dos participantes e registros inadequados ou incompletos.

Os estudos longitudinais identificaram a auto-eficácia, agressividade, uso de álcool, resistência galvânica da pele e características sociodemográficas como fatores individuais de risco para TEPT. Quanto ao contexto, foram vistas associações entre TEPT e fatores ligados à ocupação ou à vida em geral, sendo eles: tipo de formação profissional, exposição a eventos traumáticos ocupacionais e não ocupacionais e a eventos de vida estressantes (Tabela 2).

Vale mencionar que os resultados apresentados são coerentes com os preditores de TEPT estudados na população em geral. Cita-se a meta-análise de Ozer et al. ${ }^{9}$ que tem como base 68 artigos, os quais evidenciaram os seguintes fatores: exposição a eventos traumáticos no passado; capacidade de adaptação do indivíduo; história familiar de morbidade psiquiátrica; ameaça de vida percebida; suporte social e sintomas peritraumáticos.

No entanto, é preciso analisar esses dados com cautela, pois fatores de contexto mostraram-se relevantes. As diferentes prevalências em profissionais e em voluntários envolvidos em um mesmo evento catastrófico sugerem que bombeiros e socorristas não são grupos comparáveis à população geral ${ }^{28}$. É possível que o grau de experiência profissional e a construção de estratégias coletivas de enfrentamento sejam explicações plausíveis para as diferenças encontradas quando o âmbito ocupacional integra as hipóteses investigativas. Ademais, os resultados obtidos até o momento indicam a relevância de estudos em amostras específicas de trabalhadores para a investigação da exposição indireta a eventos traumáticos e suas conseqüências para a saúde mental ${ }^{7,35}$.

Há diferentes padrões de progressão de resposta a eventos traumáticos em função de características psicológicas individuais (agressividade e auto-eficácia). Não seria, 
portanto, demasiado supor que indivíduos mais susceptíveis (dadas as referidas características) parecem sofrer um efeito adverso progressivo em função da exposição. Por outro lado, aqueles considerados como menos susceptíveis não vivenciaram aumento na intensidade de sintomas da doença com o passar do tempo ${ }^{23}$. Futuros estudos poderão explorar os mecanismos de adaptação ao ambiente permeado por exposição a eventos traumáticos durante a atuação profissional em serviços de emergências.

Os resultados obtidos pelos autores evidenciaram relação entre TEPT e morbidade psiquiátrica identificada em quatro entre os seis estudos que exploraram os aspectos individuais implicados no desfecho em questão. Sintomas físicos e outras doenças também foram relatados por indivíduos com sintomas de TEPT (3/6). Tais evidências reforçam os modelos que indicam o caráter multidimensional do processo saúdedoença nos seres humanos ${ }^{7,36}$.

Os estudos realizados na Austrália aportaram aclarações importantes ao identificar o peso tanto da duração quanto da repetição da exposição a eventos traumáticos ${ }^{19,20}$. Sugere-se assim a existência de um efeito cumulativo da exposição a eventos traumáticos ocupacionais. Vale mencionar o suporte social como fator de proteção para os trabalhadores expostos ${ }^{30}$.

A exposição a eventos traumáticos foi associada ao TEPT na maioria dos estudos transversais (5/6). Os resultados indicaram que não haveria diferenças na magnitude dos sintomas a depender do tipo de exposição (direta e indireta), mas sim nos padrões em que a doença se apresenta ${ }^{29}$. Os achados corroboram o peso da exposição indireta como um fator desencadeante e a necessidade de diferenciação entre TEPT primário e secundário para a compreensão da doença.

Ressalta-se a associação encontrada entre as condições de trabalho em serviços de emergência e TEPT, já mencionada na revisão de Regher \& Bober ${ }^{7}$. Vale ainda ressaltar que, apesar de apenas cinco estudos da presente revisão terem investigado variáveis relacionadas diretamente ao trabalho como possíveis fatores de risco para TEPT (5/17), a maioria obteve resultados positivos (4/5). Além disso, a elevada prevalência de reações ao estresse grave e problemas de adaptação em trabalhadores brasileiros suscita investigações visando aprofundamento do tema ${ }^{18}$.

A evolução dos parâmetros para a confirmação do diagnóstico também é um ponto importante a ser considerado na relação entre eventos traumáticos e TEPT. Os artigos examinados consideraram critérios bastante distintos para o diagnóstico da doença. Outrora, para a terceira versão do $\mathrm{DSM}^{37}$, e sua revisão apresentada em $1987^{38}$, a definição de exposição a eventos traumáticos deveria seguir um critério objetivo. $\mathrm{Na}$ atualidade, a classificação apresentada no DSM-IV-TR admite que o evento seja definido de acordo com a percepção dos sujeitos. Quanto a isso, critica-se o caráter pouco objetivo da referida definição $0^{39,40}$.

\section{Conclusão}

Variáveis individuais e de contexto são considerados fatores associados ou de risco para TEPT e parecem explicar dimensões diferentes de adoecimento em trabalhadores. Como esperado, a exposição a eventos traumáticos é o aspecto de contexto mais investigado nos artigos examinados, indicando associações positivas com a doença. Apesar disso, a definição de evento traumático ainda é tema de muita controvérsia na literatura. Aspectos ocupacionais também são essenciais para o entendimento do TEPT em trabalhadores. Os resultados indicaram ainda que o TEPT secundário é um construto relevante, considerando os diferentes padrões de adoecimento em função da natureza da exposição. Por fim, modelos teóricos explicativos que abarquem os diferentes aspectos do TEPT em profissionais de emergência são necessários para o entendimento do fenômeno. 


\section{Referências}

1. Corneil W, Beaton R, Murphy S, Johnson C, Pike K. Exposure to Traumatic Incidents and Prevalence of Posttraumatic Stress Disorders in Urban Firefighters in Two Countries. J Occup Health Psychol 1999; 4: 131-41.

2. Van der Ploeg E, Kleber RJ Acute chronic job stressors among ambulance personnel: predictors of health symptoms. J Occup Environ Med 2003; 60: 40-6.

3. Jonsson A, Segesten K, Mattsson B. Posttraumatic stress among Swedish ambulance personnel. J Emerg Med 2003; 20: 79-84.

4. Perrin, M A, DiGrande L, Wheeler K, Thorpe L, Farfel M, Brackbill R. Differences in PTSD Prevalence and Associate Risk Factors Among World Trade Center Disaster Rescue and Recovery Workers. Am J Psychiatry 2007; 164: 1385-94.

5. Figley CR. Compassion Fatigue: coping with secondary traumatic stress in those who treat the traumatized. New York: Brunner/Mazel; 1995.

6. Associação Americana de Psiquiatria. DSM-IV-TR, Manual Diagnóstico e Estatístico dos Transtornos Mentais (4a Ed. Texto Revisado). Porto Alegre: ArtMed. (Originalmente publicado em 2000); 2002.

7. Regehr C, Bober T. In the Line of Fire-Trauma in the Emergency Services. New York: Oxford University Press; 2005.

8. Breslau N, Kessler RC. The Stressor Criterion in DSM-IV Posttraumatic Stress Disorder: An Empirical Investigation. Biol Psychiatry 2001; 50: 699-704.

9. Ozer EJ, Best SR, Lipsey TL, Weiss DS. Predictors of Posttraumatic Stress Disorder and Symptons in Adults: A Meta-Analysis. Psychol Bull 2003; 129: 52-73.

10. Brewin CR, Holmes EA. Psychological theories of posttraumatic stress disorder. Psychol Bull 2003; 23: 33976.

11. Mauer MP, Cummings KR, Carlson GA. Health Effects in New York State Personnel Who Responded to the World Trade Center Disaster. J Occup Environ Med 2007; 49: 1197-205.

12. Cetin M, Kose S, Ebrinc S, Yigit S, Elhai JD, Basoglu C. Identification and Posttraumatic Stress Disorder Symptons in Rescue Workers in the Marmara, Turkey, Earthquake. J Trauma Stress 2005; 18: 485-9.

13. Clohessy S, Ehlers A. PTSD symptoms, response to intrusive memories and coping in the ambulance service workers. Br J Clin Psychol 1999; 38: 251-65.

14. Laposa JM, Alden LE. Posttraumatic stress disorder in the emergency room: exploration of a cognitive model. Behav Res Ther 2003; 41: 49-65.
15. Bennett P, Williams Y, Page N, Hood K, Woolard M, Vetter $\mathrm{N}$. Associations between organizational and incident factors and emotional distress in emergency ambulance personnel. Br J Clin Psychol 2005; 44: 215-26.

16. Sterud T, Ekeberg O, Hem E. Health status in the ambulance services: a systematic review. BMC Health Serv Res 2006; 6: 82.

17. Regehr C, Goldberg G, Hughes J. Exposure to Human Tragedy, Empathy, and Trauma in Ambulance Paramedics. Am J Orthopsychiatry 2002; 72: 505-13.

18. Ministério da Previdência Social / Empresa de Tecnologia e Informação da Previdência Social/Instituto Nacional do Seguro Social. Anuário Estatístico da Previdência Social - 2008. Disponível em http:/ / www. previdencia.gov.br [Acessado em 24 de janeiro de 2010]

19. Bryant RA, Guthrie RM. Maladaptive self-appraisals before trauma exposure predict posttraumatic stress disorder. J Consul Clin Psychol 2007; 75: 812-5.

20. Bryant RA., Guthrie RM. Maladaptive Appraisals as a Risk Factor for Posttraumatic Stress - A Study of Trainee Firefighters. Psychol Sci 2005; 16: 749-52.

21. Guthrie RM, Bryant RA. Auditory Startle Response in Firefighters Before and After Trauma Exposure. Am J Psychiatry 2005; 162: 283-90.

22. Su TP, Lien TC, Yang CY, Su YL, Wang JH, Tsai SL et al. Prevalence of psychiatric morbidity and psychological adaptation of the nurses in a structured SARS caring unit during outbreak: A prospective and periodic assessment study in Taiwan. J Psychiatric Res 2007; 41: 119-30

23. Heinrichs M, Wagner D, Schoch W, Soravia LM, Ehlert U. Predicting Posttraumatic Stress Symptoms From Pretraumatic Risk Factors: A 2-Year Prospective FollowUp Study in Firefighters. Am J Psychiatry 2005; 162: 227686.

24. Slottje P, Witteveen AB, Twisk JWR, Smidt N, Huizink,AC, Mechelen W et al. Post-disaster physical symptoms of firefighters and police officers: role of types of exposure and Posttraumatic stress symptons. Br J Health Psychol 2008; 13: 327-42.

25. Witteveen AB, Bramsen I, Twisk JWR, Huizink AC, Slottje P, Smid T et al. Psychological Distress of Rescue Workers Eight and One-Half Years After Professional Involvement in the Amsterdam Air Disaster. J Nerv Ment Dis 2007; 195: 31-40.

26. Huizink AC, Slottje P, Witteveen AB, Bijlsma JA, Twisk JWR, Smidt N et al. Long term health complains following the Amsterdam Air Disaster in police officers and fire-fighters. Occup Environ Med 2006; 63: 657-62. 
27. van der Velden PG, Christiaanse B, Kleber RJ, Marcelissen FGH, Dorresteijn SAM, Drogendijk AN et al. The effects of Disaster exposure and post-disaster critical incidents on intrusions, avoidance reactions and health problems among firefighters: a comparative study. Stress Trauma Crisis 2006; 9: 73-93.

28. Hagh-Shenas, H, Goodarzi MA., Dehbozorgi G, Farashbandi H. Psychological Consequences of the Bam Earthquake on Professional and Nonprofessional Helpers. J Trauma Stress 2005; 18: 477-83.

29. Alden LE, Regambal MJ, Laposa JM. The effects of direct versus witnessed threat on emergency department healthcare workers: Implications for PTSD Criterion A. J Anxiety Disord 2008; 22: 1337-46.

30. Mitani S. Comparative Analysis of the Japanese Version of the Revised Impact of Event Scale: a study of firefighters. Prehosp Disaster Med 2008; 23: 20-6.

31. Berger W, Figueira I, Maurat AM, Bucassio EP, Vieira I, Jardim SR et al. Partial and Full PTSD in Brazilian Ambulance Workers: Prevalence and Impact on Health and Quality of Life. J Trauma Stress 2007; 20: 637-42.

32. Chen YS, Chen MC, Chou FHC, Sun FC, Chen PC, Tsai $\mathrm{KY}$ et al. The relationship between quality of life and posttraumatic stress disorder or major depression for firefighters in Kaohsiung, Taiwan. Qual Life Res 2007;16: 1289-97.

33. Del Ben KS, Scotti JR, Chen YC, Fortson BL. Prevalence of posttraumatic stress disorder symptons in firefighters. Work \& Stress 2006; 20: 37-48.
34. Nortje C, Roberts CB, Möller AT. Judgment of risk in traumatized and non-traumatized emergency medical service personnel. Psychol Rep 2004; 95: 1119-28.

35. Wagner D, Heinrichs M Ehlert U. Prevalence of Symptons of Posttraumatic Stress Disorder in German Professional Firefighters. Am J Psychiatry 1998; 155: 1727-32

36. Roberts AR. Crisis intervention handbook: assessment, treatment, and research. New York: Oxford University Press; 2000.

37. American Psychiatry Association (1980). DSM-III, Diagnostic and Statistical Manual of Mental Disorders (3 ${ }^{\text {a }}$ Ed.). Washington, DC: Autor.

38. American Psychiatry Association (1987). DSM-III-R, Diagnostic and Statistical Manual of Mental Disorders

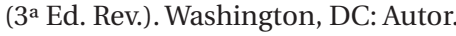

39. Rosen GM, Lilienfeld SO. Posttraumatic stress disorder: an empirical evaluation of core assumptions. Clin Psychol Rev 2008; 28: 837-68.

40. McNally RJ. Progress and Controversy in the Study of Posttraumatic Stress Disorder. Ann Rev Psychol 2003; 54: 229-52.

Recebido em: 07/02/10

Versão final reapresentada em: 28/10/10 Aprovado em: 12/11/10 